\title{
Defects in amorphous phase-change materials
}

\author{
Jennifer Luckas \\ I. Physikalisches Institut, RWTH Aachen University, 52056 Aachen, Germany; and Laboratoire de Génie Electrique \\ de Paris (CNRS UMR 8507), Supelec, Universités Paris VI et XI, Plateau de Moulon, 91190 Gif sur Yvette, France
}

Daniel Krebs

IBM Zürich Research Laboratory, 8803 Rüschlikon, Switzerland

Stephanie Grothe

I. Physikalisches Institut, RWTH Aachen University, 52056 Aachen, Germany

Josef Klomfaß and Reinhard Carius

IEF-5 Photovoltaik, Forschungszentrum Jülich, 52425 Jülich, Germany

Christophe Longeaud

Laboratoire de Génie Electrique de Paris (CNRS UMR 8507), Supelec, Universités Paris VI et XI, Plateau de Moulon, 91190 Gif sur Yvette, France

Matthias Wuttig ${ }^{\text {a) }}$

I. Physikalisches Institut, RWTH Aachen University, 52056 Aachen, Germany; and JARA Fundamentals of Future Information Technology, RWTH Aachen University, 52056 Aachen, Germany

(Received 30 August 2012; accepted 12 March 2013)

Understanding the physical origin of threshold switching and resistance drift phenomena is necessary for making a breakthrough in the performance of low-cost nanoscale technologies related to nonvolatile phase-change memories. Even though both phenomena of threshold switching and resistance drift are often attributed to localized states in the band gap, the distribution of defect states in amorphous phase-change materials (PCMs) has not received so far, the level of attention that it merits. This work presents an experimental study of defects in amorphous PCMs using modulated photocurrent experiments and photothermal deflection spectroscopy. This study of electrically switching alloys involving germanium $(\mathrm{Ge})$, antimony $(\mathrm{Sb})$ and tellurium $(\mathrm{Te})$ such as amorphous germanium telluride (a-GeTe), $\mathrm{a}-\mathrm{Ge}_{15} \mathrm{Te}_{85}$ and $\mathrm{a}-\mathrm{Ge}_{2} \mathrm{Sb}_{2} \mathrm{Te}_{5}$ demonstrates that those compositions showing a high electrical threshold field also show a high defect density. This result supports a mechanism of recombination and field-induced generation driving threshold switching in amorphous chalcogenides. Furthermore, this work provides strong experimental evidence for complex trap kinetics during resistance drift. This work reports annihilation of deep states and an increase in shallow defect density accompanied by band gap widening in aged a-GeTe thin films.

\section{INTRODUCTION}

Phase-change materials (PCMs) exhibit a combination of extraordinary properties enabling their application in information storage. ${ }^{1}$ First, these materials show a drastic difference in resistivity or reflectivity between amorphous and crystalline phases. ${ }^{2,3}$ In addition, this class of materials exhibits very fast crystallization kinetics proceeding on a submicrosecond time scale. In some alloys such as germanium telluride $(\mathrm{GeTe})$ the phase transitions can even be as fast as $1 \mathrm{~ns} .{ }^{4}$ Hence, PCMs offer the possibility to create a universal memory combining nonvolatility with dynamic random access memory (DRAM)-like switching speeds. ${ }^{5,6}$ The successful application of PCM in electronic devices is

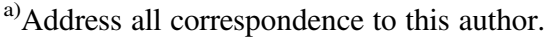
e-mail: wuttig@physik-rwth-aachen.de This paper has been selected as an Invited Feature Paper. DOI: $10.1557 / \mathrm{jmr} .2013 .72$
}

made possible by the remarkable nonlinear I-V characteristics observed in the amorphous state. ${ }^{1,7}$ The electrical resistivity decreases significantly by several orders of magnitude by applying an electric field exceeding a materialdependent critical value typically of $10 \mathrm{~V} / \mu \mathrm{m} .{ }^{8}$ This sudden breakdown in resistivity is known as the threshold switching effect and the physics behind that phenomenon is still a matter of research. Below this threshold the amorphous state resistivity shows a thermally activated behavior and is observed to increase with aging duration. Furthermore, the increase in resistivity accelerates with annealing temperature. ${ }^{9}$ To realize the multilevel storage potential of the PCM technology, it is crucial to identify materials with a stable amorphous state resistivity. Finding suitable materials could result in a massive increase in storage density. Binary phase-change alloy GeTe offers both very fast crystallization kinetics, and a high crystallization temperature of $180{ }^{\circ} \mathrm{C}$, which is attractive for industrial applications. ${ }^{10}$ However, the drift of the amorphous state 
resistivity in amorphous GeTe (a-GeTe) is rather high. ${ }^{11}$ $\mathrm{Ge}_{15} \mathrm{Te}_{85}$, on the contrary shows much slower crystallization kinetics on a timescale of $100 \mu \mathrm{s},{ }^{10}$ but a more stable amorphous state.

Even though many models attribute the threshold switching and resistance drift effects to trap kinetics, ${ }^{12-16}$ little is known about the defect distribution in amorphous phase-change alloys. As the physical properties can be drastically varied with the proportion of tellurium (Te) content in binary GeTe alloys, this study compares traps state densities of a-Ge ${ }_{15} \mathrm{Te}_{85}$ to two common PCMs a-GeTe and $\mathrm{a}-\mathrm{Ge}_{2} \mathrm{Sb}_{2} \mathrm{Te}_{5}$ with the objective of gaining a better insight into the mechanisms underlying the effects of threshold switching. To get a better insight of resistance drift phenomena the evolution of defect state densities, activation energies of electronic conduction and optical band gaps have been systematically studied in aged and postannealed a-GeTe thin films.

\section{EXPERIMENTAL TECHNIQUES USED TO STUDY RESISTANCE DRIFT PHENOMENA AND DEFECT STATE DENSITIES IN AMORPHOUS PHASE-CHANGE MATERIAL THIN FILMS}

\section{A. Sample preparation}

The thin films studied were produced by direct current (dc) magnetron sputtering using a LS 320 von Ardenne system (Dresden, Germany) and stoichiometric targets of $99.99 \%$ purity. The chalcogenide thin films were deposited on substrates meeting the specific requirement of subsequent measurements adjusting the sputtering power to $20 \mathrm{~W}$, the background pressure to $10^{-6} \mathrm{mbar}$, and the argon gas pressure to $5 \times 10^{-3}$ mbar. X-ray measurements under grazing incidence revealed that all samples were amorphous after deposition. The thickness of the chalcogenide films was determined from known sputter rates determined in advance for each target. For all films the film thickness was verified using spectroscopic ellipsometry measurements. Both methods gave consistent values for the film thickness $d$ chosen to be $\sim 200 \mathrm{~nm}$ in this study unless stated otherwise.

\section{B. Electrical characterization of resistance drift}

For investigating the temporal drift of the amorphous state resistivity a sophisticated set-up has been developed. The system is based on the four-point van der Pauw method. Electrical van der Pauw experiments enable the measurement of sheet resistance $R$, which is directly related to the resistivity according to $\rho=R \cdot d$. To realize the required four-point geometry at first four aluminum contacts were sputtered symmetrically on solvent-cleaned $2 \times 2 \mathrm{~cm}^{2}$ glass substrates. Subsequently, a chalcogenide thin film (size $1 \times 1 \mathrm{~cm}^{2}$ ) was sputter-deposited centered with respect to the electrodes. A heated sample stage enables study of the change of resistivity during heating. For avoid- ing oxidation effects these measurements were performed under argon atmosphere.

\section{Photothermal deflection spectroscopy}

Photothermal deflection spectroscopy (PDS) is based on the mirage effect. ${ }^{17}$ The sample under study is surrounded by a deflection medium while a monochromatic light source illuminates the sample surface. Due to recombination in available states the material under study heats the deflection medium near the sample surface creating a thermal lens, i.e., a gradient in refractive index, deflecting a laser passing near the sample surface. The deflection angle contains information on the density of defect states within the band gap. In the present study carbon tetrachloride $\left(\mathrm{CCl}_{4}\right)$ was used as the deflection medium. Monochromatized light from halogen and xenon lamps was used to illuminate a-GeTe thin films. The intensity of the incoming and transmitted light has been detected as well as the deflection angle of a semiconductor laser passing the sample surface at a distance of approximately $30 \mu \mathrm{m}$. From these data taken at room temperature, the absorption coefficient $\alpha$ at different photon energies $E$ has been calculated knowing the film thickness $d$ and variation of the refraction index $n(E)$ near the optical band gap $E_{\mathrm{g}}$ of the studied a-GeTe thin films to estimate the absorbed light intensity. For achieving a good balance between transmission and reflection in the experiments a film thickness of $500 \mathrm{~nm}$ has been chosen.

\section{Modulated photocurrent experiments}

Modulated photocurrent (MPC) experiments enable a spectroscopy of the defect state density within the band gap. Originally this method has been developed to study photovoltaic materials. ${ }^{18-20}$ Recently, the MPC technique has been applied successfully to amorphous PCMs - a class of chalcogenide materials which generally shows a very low photoconductivity at room temperature. ${ }^{21}$ Generally chalcogenide glasses show n-type conductivity in Hall effect measurements, whereas these alloys demonstrate p-type conductivity in thermoelectric Seebeck experiments. A similar phenomenon is also observed in other disordered materials such as hydrogenated amorphous silicon and is commonly known as the anomalous Hall effect. At this point one should recall that electric and magnetic fields may lead to complex scattering mechanisms resulting in ordinary and anomalous contributions to the Hall conductivity. Whereas the ordinary Hall conductivity mainly depends on the carrier type and concentration, different physical mechanisms may contribute to a nonzero anomalous Hall conductivity. These scattering mechanisms are expected to depend strongly on disorder. ${ }^{22}$ Thermoelectric measurements are based on carrier diffusion induced by temperature gradients. Hence, a thermoelectric approach is 
expected to give a reliable result for amorphous chalcogenides and consequently p-type conductivity is assumed in this work.

The MPC technique exploits the interaction of free holes with localized hole trap states to gain information about the density of states within the band gap. In these experiments the sample surface is illuminated by a modulated light flux $F$ :

$$
F=F_{\mathrm{dc}}+F_{\mathrm{ac}} \sin (\omega t) .
$$

This periodic excitation gives rise to the creation of photoexcited holes induced by band-to-band transitions. However, localized defect states within the band gap capture free holes from the valence band. As long as direct carrier transitions between different localized defect states can be neglected, trapped holes do not contribute to photoconductivity. Nevertheless, captured holes can be thermally released out of the trap toward extended states beyond the valence band edge. As a consequence of these multiple trapping and release processes visualized in Fig. 1, a phase lag $\phi$ exists between the hole-driven photocurrent $I$ and the excitation flux $F$ :

$$
I=I_{\mathrm{dc}}+I_{\mathrm{ac}} \sin (\omega t+\phi) .
$$

Multiple trapping involves all localized traps within the band gap as illustrated in Fig. 1. However, it has been shown that the photocurrent is dominated by those holes that are trapped and released at the same frequency as the frequency of the light modulation. Hence, only those traps having an emission rate $e_{p}(E, T)$ equal to the excitation frequency $\omega$ contribute significantly to the phase shift $\phi$. Solving the corresponding rate equations under the assumptions that the alternating photocurrent $I_{\mathrm{ac}}$ is mainly driven by holes and that multiple trapping processes dominate the electronic transport, the reduced defect

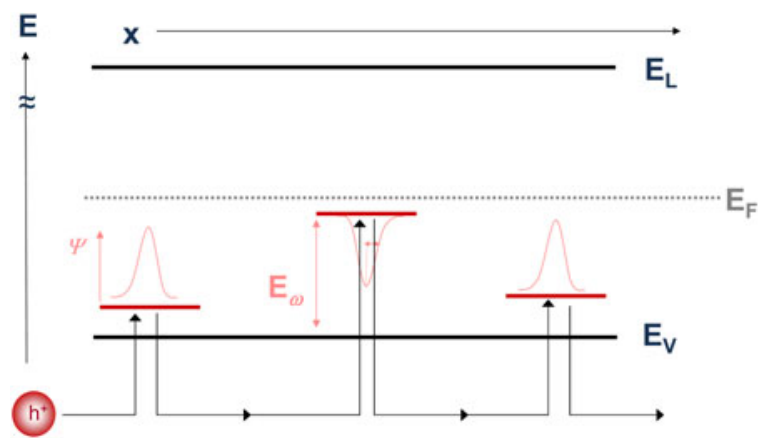

FIG. 1. Hole trap states within the band gap (indicated by red lines and localized wave functions $\Psi$ ) capture free holes from the valence band. After a certain time a hole trapped at $E_{\omega}$ is thermally released back to the valence band. These trapping and release processes repeat continuously. This interrupted extended state transport is denoted as multiple trapping transport for which the basic MPC formulas are derived by solving the corresponding rate equations, see Ref. 18-20. density $N c / \mu$ can be determined by the following relation ${ }^{19,20}$ :

$$
\frac{c_{p} N\left(E_{\omega}\right)}{\mu_{p}}=\frac{2}{\pi k_{b} T} A q \varepsilon_{\mathrm{el}} \cdot g_{\mathrm{ac}} \cdot \frac{\sin (\phi)}{\left|I_{\mathrm{ac}}\right|},
$$

where $N$ is the density of states, $c_{p}$ is the capture coefficient describing the interaction between localized trap states and free holes, $\mu_{p}$ is the free hole mobility, $A$ denotes the conduction cross-section, $q$ is the absolute value of the electronic charge, $k_{\mathrm{B}}$ denotes the Boltzmann constant, $\varepsilon_{\mathrm{el}}$ is the applied electric field, and $g_{\text {ac }}$ is the ac generation rate of photo carriers. All parameters of the right hand side including the phase shift $\phi$ and the amplitude of the alternating photocurrent $\left|I_{\mathrm{ac}}\right|$ can be obtained directly from experiment.

The energy scaling of the reduced density $N\left(E_{\omega}\right) c / \mu$ is defined by the fact that only those hole traps whose emission rate $e_{p}$ equals the excitation frequency $\omega$ contribute significantly to a nonzero phase shift $\phi$. Thus the relation $e_{p}\left(E_{\omega}\right)=v_{p} \cdot \exp \left[-E_{\omega} /\left(k_{\mathrm{b}} T\right)\right]=\omega$ leads to the classical energy scaling valid for all p-type materials, such as a-GeTe, a- $\mathrm{Ge}_{15} \mathrm{Te}_{85}$ and $\mathrm{a}-\mathrm{Ge}_{2} \mathrm{Sb}_{2} \mathrm{Te}_{5}{ }^{19,20}$

$$
E_{\omega}=E-E_{\mathrm{v}}=k_{\mathrm{b}} T \ln \left(v_{p} / \omega\right),
$$

where $v_{p}$ is the attempt-to-escape frequency of a hole trapped in a defect state located at energy $E_{\omega}$. According to Eqs. (3) and (4) a simple spectroscopy of the quantity $N c / \mu$ within the band gap is possible by measuring couples $\left(\phi,\left|I_{\mathrm{ac}}\right|\right)$ at different excitation frequencies $\omega$ and temperatures $T$. Figure 2 illustrates the probed energy ranges for two different temperatures $T_{1}<T_{2}$ by varying the modulation frequency $\omega$ between $\omega_{\min }$ and $\omega_{\max }$ - the set-up limited $\omega$ range used in the experiment. As illustrated in Fig. 2 hole traps near to the valence band edge are probed at low temperatures and high modulation frequency $\omega$, whereas hole traps deep into the gap are measured at high temperatures and low excitation frequency $\omega$.

Amorphous chalcogenides generally show a strongly temperature-dependent band gap resulting in a shift of the valence band edge with temperature, i.e., $E_{\mathrm{v}}=E_{\mathrm{v}}(T)$. Consequently, MPC curves taken at different temperatures do no longer refer to the same zero point of the energy scale as assumed in the classic energy scale. Recent studies have shown that the temperature dependence of the band gap in a-GeTe and a- $\mathrm{Ge}_{2} \mathrm{Sb}_{2} \mathrm{Te}_{5}$ can be taken into account by a corrected energy scale given by ${ }^{23}$ :

$$
E_{\omega}=E-E_{\mathrm{v}}=k_{\mathrm{b}} T \ln \left(v_{p} / \omega\right)-\xi T^{2},
$$

where $\xi$ describes the parabolic decrease of the optical band gap with increasing temperature. A detailed discussion of the influence of a temperature-dependent band gap on the MPC energy scale can be found in Ref. 23. 


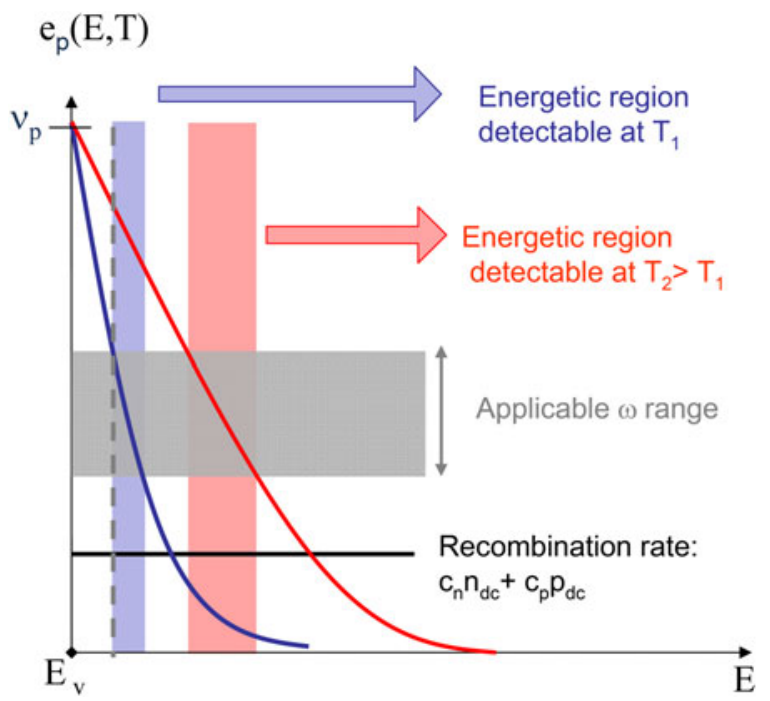

FIG. 2. In p-type materials MPC experiments probe the defect state concentration $N$ of those multiple trapping levels above the valence band edge $E_{\mathrm{v}}$, whose emission rate $e\left(E_{\omega}, T\right)$ equals the excitation frequency $\omega$ of the exciting light source, i.e., $e\left(E_{\omega}, T\right)=\omega>$ recombination rate. The two decreasing full lines represent the variations of $e(E, T)$ with $E$ for two temperatures $T_{1}$ (blue) and $T_{2}$ (red). At a given temperature $T$ states within a certain energy range can be probed using an applicable $\omega$ range (blue shaded range at $T_{1}$ and red shaded range at $T_{2}>T_{1}$ ). Hence, a systematic variation of excitation frequency $\omega$ and temperature $T$ enables a spectroscopic scan of the defect state distribution.

This study compares reduced defect state $N c / \mu$ densities of different amorphous chalcogenides. One should note that that the MPC-DOS $N c / \mu$ is of primary importance for electronic transport phenomena in semiconductors. Indeed, electronic transport phenomena are not influenced by the defect state distribution $N(E)$ alone. States with a high density $N$ but a low capture coefficient $c$ may have the same influence on trapping and recombination as trap states with a low density $N$ but a high capture coefficient $c$. This influence on the electronic transport is reflected by the $N c$ product. Furthermore, a semiconductor characterized by a low $N c$ product and a low extended state mobility will demonstrate transport properties, in terms of carrier flow or current, similar to a semiconductor having a high $N c$ product and a high mobility. Consequently the reduced density of states $N c / \mu$ is an excellent indicator of the electronic transport properties of semiconductors. Furthermore, it should be recalled at this point that Eq. (3) is only valid for defect states acting as multiple trapping centers (emission rate $>$ recombination rate) but not as recombination centers (recombination rate $>$ emission rate), the recombination rate being defined as $c_{n} n_{\mathrm{dc}}+c_{p} p_{\mathrm{dc}}$, where $c_{n}\left(c_{p}\right)$ is the capture coefficient for electrons (holes) and $n_{\mathrm{dc}}\left(p_{\mathrm{dc}}\right)$ is the free electrons (holes) concentration. Therefore, the analysis of MPC data via Eqs. (3) and (4) or Eqs. (3) and (5) enables the investigation of defect state densities within a limited energy range only, i.e., at most from the valence band edge to the dark Fermi level.
The attempt-to-escape frequency $v_{p}$ cannot be measured directly, but an order of magnitude can be estimated within the MPC analysis procedure demonstrated later in this work. Hence, MPC measurements were performed in a large temperature range, which varied in this study from 90 to $300 \mathrm{~K}$. The chalcogenide films studied have been deposited on sapphire substrates (size $1 \times 1 \mathrm{~cm}^{2}$ ) to ensure a good thermal contact on the cold finger of the cryostat during the experiment.

\section{RESULTS AND DISCUSSION}

\section{A. Defect state densities of electrical switching chalcogenides}

Recent studies have shown that the electrical threshold field is strongly material-dependent. ${ }^{24,25}$ A phasechange alloy such as $\mathrm{Ge}_{15} \mathrm{Sb}_{85}$, which possesses a small optical band gap $E_{\mathrm{g}}$ exhibits a low electrical threshold field $E_{\mathrm{t}}{ }^{16,26,27}$ However, materials with similar band gaps as a-GeTe, a- $\mathrm{Ge}_{2} \mathrm{Sb}_{2} \mathrm{Te}_{5}$ and a- $\mathrm{Ge}_{15} \mathrm{Te}_{85}$ exhibit very different threshold fields (see Table I). Consequently, the large variation in $E_{\mathrm{t}}$ ranging from $37 \mathrm{~V} / \mu \mathrm{m}$ for a- $\mathrm{Ge}_{15} \mathrm{Te}_{85}$ to $143 \mathrm{~V} / \mu \mathrm{m}$ for a-GeTe cannot be properly described by the difference in optical band gaps alone. With the aim to study the influence of localized defect states on electrical switching, this work compares defect state densities measured by MPC experiments in amorphous deposited $\mathrm{a}-\mathrm{GeTe}, \mathrm{a}-\mathrm{Ge}_{2} \mathrm{Sb}_{2} \mathrm{Te}_{5}$ and $\mathrm{a}-\mathrm{Ge}_{15} \mathrm{Te}_{85}$ thin films and relates the defect density to the threshold field.

Figure 3 presents MPC spectra using a corrected energy scale according to Eq. (5). These measurements were performed using a light-emitting diode of wave length $\lambda=850 \mathrm{~nm}$. For all measurements the amplitude of the alternating photon flux was chosen to be $40 \%$ of the continuous flux and the continuous photon flux was adjusted to $F_{\mathrm{dc}}=10^{16} /\left(\mathrm{cm}^{2} \mathrm{~s}\right)$ if not stated otherwise. Each MPC curve, defined by a symbol, is taken at a given temperature by varying the excitation frequency $f=\omega / 2 \pi$ from $12 \mathrm{~Hz}$ to $40 \mathrm{kHz}$ in such a way that that $f_{i+1}=f_{i} \times 1.5$. At a given temperature states closer to the valence band edge are probed at $40 \mathrm{kHz}$, whereas deeper states are probed at lower frequencies, see Eq. (4) or Eq. (5). Likewise, increasing the temperature $T$ enables a spectroscopy of trap states lying deeper within the gap. Thereby, the temperature steps have to be chosen sufficiently small so that the same trap state density is measured with different pairs of $(f, T)$, see Fig. 2. Consequently, the envelope of overlapping MPC curves taken at different temperatures reveals the probed density of states.

This is why the value of the attempt-to-escape frequency $v_{p}$ which maximizes the overlap of MPC curves taken at different temperatures has to be chosen to define their energetic position within the forbidden band gap. Since the attempt-to-escape frequency $v_{p}$ reflects the capture cross section of the hole trap considered, its value may differ 
TABLE I. Electrical threshold fields and optical band gaps for different amorphous deposited chalcogenides taken from the literature as compared with reduced defect state densities $N c / \mu$ describing valence band tail (VB), shallow (L1) and deep (L2) defect state densities presented in this work.

\begin{tabular}{|c|c|c|c|c|}
\hline Alloy & $\begin{array}{l}\text { Threshold } \\
\text { field }(\mathrm{V} / \mu \mathrm{m})\end{array}$ & $\begin{array}{l}\text { Band gap at } \\
300 \mathrm{~K}(\mathrm{eV})\end{array}$ & $\begin{array}{c}\text { Mid gap defect } \\
\text { state density } N c / \mu\left(/ \mathrm{cm}^{-2}{\left.\mathrm{~V} / \mathrm{eV}^{-1}\right)}^{-}\right.\end{array}$ & $\begin{array}{c}\text { Shallow defect maximum } \\
\text { density } N c / \mu\left(/ \mathrm{cm}^{-2} \mathrm{~V} / \mathrm{eV}^{-1}\right)\end{array}$ \\
\hline $\mathrm{Ge}_{15} \mathrm{Sb}_{85}$ & $08^{24}$ & $0.41^{25}$ & $\ldots$ & $\ldots$ \\
\hline AgInSbTe & $19^{24}$ & $0.63^{25}$ & & $\ldots$ \\
\hline $\mathrm{Ge}_{15} \mathrm{Te}_{85}$ & $37^{24}$ & $1.00^{25}$ & $1 \times 10^{8}(\mathrm{VB})$ & Not detected \\
\hline $\mathrm{Ge}_{2} \mathrm{Sb}_{2} \mathrm{Te}_{5}$ & $56^{24}$ & $0.80^{25}$ & $1 \times 10^{10}(\mathrm{~L} 2)$ & $4 \times 10^{9}(\mathrm{~L} 1)$ \\
\hline GeTe & $143^{23}$ & $0.81^{25}$ & $5 \times 10^{10}(\mathrm{~L} 2)$ & $1 \times 10^{10}(\mathrm{~L} 1)$ \\
\hline
\end{tabular}
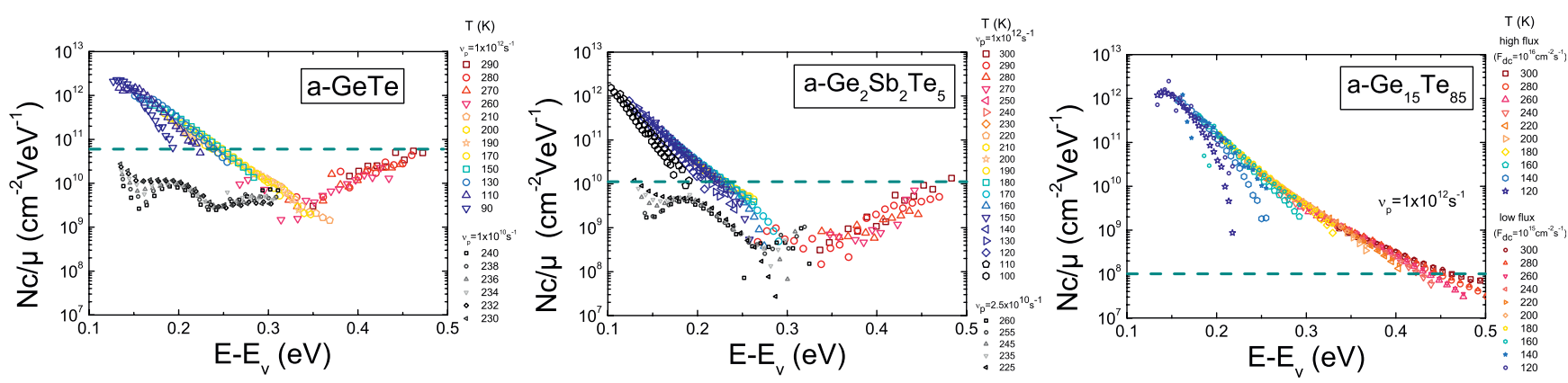

FIG. 3. Defect state densities $N c / \mu$ measured by MPC experiments in a-GeTe, a- $\mathrm{Ge}_{2} \mathrm{Sb}_{2} \mathrm{Te}_{5}$ and a-Ge $\mathrm{Ge}_{15} \mathrm{Te}_{85}$ thin films. The MPC data shown here are scaled using Eq. (5), which takes the temperature dependence of the band gap into account. Both phase-change alloys $\left(\mathrm{a}-\mathrm{GeTe}\right.$ and a-Ge $\left.\mathrm{Sb}_{2} \mathrm{Te}_{5}\right) \operatorname{show}$ three characteristic defect state distributions: valence band tail states probed from 90 to $210 \mathrm{~K}$ (VB), shallow defect states located at $\sim 0.2 \mathrm{eV}$ from the valence band edge $E_{\mathrm{v}}$ detected from 220 to $250 \mathrm{~K}$ (L1 defects) and deep defect states measured from 260 to $300 \mathrm{~K}$ (L2 defects). In contrast to a-GeTe and $\mathrm{a}-\mathrm{Ge}_{2} \mathrm{Sb}_{2} \mathrm{Te}_{5}, \mathrm{a}-\mathrm{Ge}_{15} \mathrm{Te}_{85}$ presents only valence band tail states over the whole investigated temperature range. Amorphous GeTe presents the highest density of deep (L2: $N c / \mu=5 \times 10^{10} / \mathrm{cm}^{2} \mathrm{~V} / \mathrm{eV}$ ) as well as shallow trap states (L1: $N c / \mu=1 \times 10^{10} / \mathrm{cm}^{2} \mathrm{~V} / \mathrm{eV}$ ), followed by a-Ge $\mathrm{Sb}_{2} \mathrm{Te}_{5}$ (L2: $N c / \mu=1 \times 10^{10} / \mathrm{cm}^{2} \mathrm{~V} / \mathrm{eV}$ and L1: $N c / \mu=4 \times 10^{9} / \mathrm{cm}^{2} \mathrm{~V} / \mathrm{eV}$ ). In contrast to both PCMs a- $\mathrm{Ge}_{15} \mathrm{Te}_{85}$ shows no L1 or L2 defect distributions and valence band tail densities are measured down to $N c / \mu=10^{8} / \mathrm{cm}^{2} \mathrm{~V} / \mathrm{eV}$ close to mid gap.

significantly for different defect state distributions such as band tails, shallow or deep defects.

Both phase-change alloys a-GeTe and $\mathrm{a}-\mathrm{Ge}_{2} \mathrm{Sb}_{2} \mathrm{Te}_{5}$ show three different characteristic defect state distributions: valence band tail states (VB states), shallow defects (L1 states) and deep defects (L2 states). Valence band tail states were probed from 90 to $210 \mathrm{~K}$. The MPC curves describing the valence band tail show the highest overlap adjusting the attempt-to-escape frequency to $v_{p}=10^{12} / \mathrm{s}$. However, some MPC curves describing the valence band tail distribution depart significantly from the common envelope especially at low excitation frequencies of the modulation flux, see Fig. 3. This departure from the envelope also referred as MPC "tails" is well known in the literature and results from the impact of recombination. The recombination zone is energetically located around the dark Fermi level and its width increases with increasing light flux and decreasing temperature. If the probed states lie energetically in the recombination zone those probed trap states do not act as multiple trapping centers and an analysis of MPC data according to Eq. (3) — valid for multiple trapping centers only-leads to erroneous results. Consequently, these MPC "tails" are not representative of the probed DOS but rather depend on the DOS multiplied by its occupation function, where the latter evolves with the temperature at which the MPC experiment is performed. At very low temperatures MPC 'tails' may also result from an increasing influence of hopping conduction. ${ }^{28}$

In a temperature range from 220 to $250 \mathrm{~K}$ shallow defect states are probed. However, these shallow L1 states cannot be properly described by $-v_{p}=10^{12} / \mathrm{s}$. Considering the influence of a temperature-dependent band gap to calculate their energetic position with respect to the valence band edge $E_{\mathrm{v}}$, [see Eq. (5)] the attemptto-escape frequency has to be chosen equal to $v_{p}=1 \times 10^{10} / \mathrm{s}$ for ensuring a good overlap of these MPC curves taken from 220 to $250 \mathrm{~K}$. Furthermore, a-GeTe and a- $\mathrm{Ge}_{2} \mathrm{Sb}_{2} \mathrm{Te}_{5} \mathrm{dem}-$ onstrate the presence of a deep defect distribution measured from 260 to $300 \mathrm{~K}$. These deep L2 defects are scaled with $v_{p}=10^{12} / \mathrm{s}$.

In contrast to the above two PCMs, the Te-rich alloy a- $\mathrm{Ge}_{15} \mathrm{Te}_{85}$ shows the existence of valence band tail states over the whole investigated temperature range investigated from 120 to $300 \mathrm{~K}$. Even with a lower flux $F_{\mathrm{dc}}=10^{15} /\left(\mathrm{cm}^{2} \mathrm{~s}\right)$-leading to a lower splitting of both quasi-Fermi levels limiting the width of the recombination zone $\mathrm{e}^{29,30}$-no additional defect state distributions could be detected.

The MPC spectra illustrated in Fig. 3 demonstrate the reduced density of states $N c / \mu$ from the valence band 
$E_{\mathrm{v}}$ edge to the dark Fermi level, which is expected to lie close to mid gap in amorphous chalcogenides. ${ }^{31,32}$ Thus a-GeTe shows the highest density of deep L2 defect

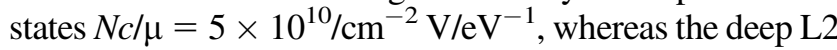
defect state density in a-Ge $\mathrm{Sb}_{2} \mathrm{Te}_{5}$ is significantly lower, i.e., $N c / \mu=1 \times 10^{10} / \mathrm{cm}^{2} \mathrm{~V} / \mathrm{eV}$ [indicated by the horizontal dashed lines in Figs. 3(a) and 3(b)]. The good glass-former a- $\mathrm{Ge}_{15} \mathrm{Te}_{85}$ shows the lowest trap state deep into the forbidden energy gap, i.e., $N c / \mu(\mathrm{VB})=1 \times 10^{8} / \mathrm{cm}^{2} \mathrm{~V} / \mathrm{eV}$. Furthermore, a-GeTe shows the highest density of shallow L1 trap states reaching a maximum value of $N c / \mu(0.2 \mathrm{eV})=1 \times 10^{10} / \mathrm{cm}^{2} \mathrm{~V} / \mathrm{eV}$. In comparison to a-GeTe a- $\mathrm{Ge}_{2} \mathrm{Sb}_{2} \mathrm{Te}_{5}$ shows a lower density of shallow $\mathrm{L} 1$ traps, i.e., $N c / \mu(0.2 \mathrm{eV})=4 \times 10^{9} / \mathrm{cm}^{2} \mathrm{~V} / \mathrm{eV}$. In contrast to both phase-change alloys no shallow L1 or deep L2 defects could be detected in a- $\mathrm{Ge}_{15} \mathrm{Te}_{85}$.

These results, summarized in Table I, underline that not only a wide band gap but also a high density of deep and shallow states $N c / \mu$ in addition to their valence band tail states promote a high electrical threshold field $E_{\mathrm{t}}$. This finding supports a generation-recombination model for electrical threshold switching in amorphous chalcogenides originally proposed by Adler. ${ }^{33}$ In this model fieldinduced generation of carriers over the band gap-such as impact ionization ${ }^{34}$-has to outbalance their recombination in localized defects to fulfill the switching condition. Consequently, this model predicts an increasing threshold field with increasing optical band gap or increasing reduced defect states density $N c / \mu$.

Figure 4 compares the temporal drift of the amorphous state resistivity measured in the three alloys a-GeTe, $\mathrm{a}-\mathrm{Ge}_{2} \mathrm{Sb}_{2} \mathrm{Te}_{5}$ and $\mathrm{a}-\mathrm{Ge}_{15} \mathrm{Te}_{85}$ at $50{ }^{\circ} \mathrm{C}$. In all the three chalcogenide alloys, the evolution of the amorphous state resistivity at $50{ }^{\circ} \mathrm{C}$ is properly described by the power law:

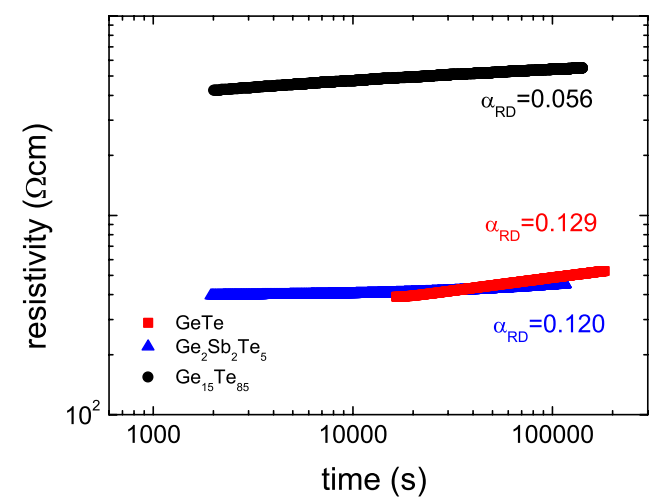

FIG. 4. Evolution of the amorphous state resistivity measured during annealing chalcogenide thin films at $50{ }^{\circ} \mathrm{C}$. For a better data visualization the experimental time scale is shifted by $t_{\mathrm{s}}$, see Eq. (6). In all three alloys, this evolution of the amorphous state resistivity is correctly described by a power law. The drift exponent $\alpha_{\mathrm{RD}}$ is observed to be rather high in both investigated phase-change alloys a-GeTe $\left(\alpha_{\mathrm{RD}}=0.129\right)$ and a- $\mathrm{Ge}_{2} \mathrm{Sb}_{2} \mathrm{Te}_{5}\left(\alpha_{\mathrm{RD}}=0.120\right)$ indicating a strong drift in resistivity. In comparison a- $\mathrm{Ge}_{15} \mathrm{Te}_{85}$ shows a much lower value $\alpha_{\mathrm{RD}}=0.056$ and thus a lower drift in resistivity.

$$
\rho=\rho_{0}\left(t+t_{\mathrm{s}}\right)^{\alpha \mathrm{RD}} .
$$

The parameter $\rho_{0}$ denotes the resistivity measured at the onset of the drift experiment. The parameter $t_{\mathrm{s}} \neq 0$ illustrates that the starting point of the measurement defined as $t=0 \mathrm{~s}$ does not match with the beginning of the drift mechanism of the amorphous deposited thin film, which should be expected to start already during or at least directly after completion of the deposition process. The change of the amorphous state resistivity over time is governed by the drift coefficient $\alpha_{\mathrm{RD}}$, i.e., the stronger the drift the higher the value of $\alpha_{\mathrm{RD}}$. Consequently, the resistance drift in both phase-change alloys GeTe $\left(\alpha_{\mathrm{RD}}=0.129\right)$ and $\mathrm{a}-\mathrm{Ge}_{2} \mathrm{Sb}_{2} \mathrm{Te}_{5}$ $\left(\alpha_{\mathrm{RD}}=0.120\right)$ is rather strong when compared with $\mathrm{a}-\mathrm{Ge}_{15} \mathrm{Te}_{85}\left(\alpha_{\mathrm{RD}}=0.056\right)$. However, in contrast to both PCMs, a- $\mathrm{Ge}_{15} \mathrm{Te}_{85}$ shows only valence band tail states and no additional shallow or deep defect state distributions. These large differences in resistance drift phenomena and defect state densities raise the question of the impact of localized defects states on resistance drift phenomena. This point is addressed in Sec. III. B.

\section{B. Evolution in defect state density in aged a-GeTe thin films}

The impact of localized defect states on resistance drift phenomena has been discussed inconclusively and contradictingly in the literature. Whereas some authors propose a structural relaxation of the glassy state leading to defect annihilation, ${ }^{15,35}$ there are other authors who claim an increasing defect state distribution with time. ${ }^{14}$

In this section, the temporal evolution of the defect state density in a-GeTe thin films is studied to identify a possible link between trap kinetics and resistance drift. Figure 3 presents MPC spectra taken on the same a-GeTe thin film for different sample ages. To facilitate a better interpretation of data both spectra in Fig. 5 are scaled according to Eq. (5) like all spectra in Fig. 3.

Aging demonstrates to have a significant influence on the measured MPC spectra, in particular on the defect levels. With increasing sample age a lower density of deep states is observed as predicted by Ielmini. ${ }^{15}$ At a sample age of 29 days the MPC curves taken from 260 to $290 \mathrm{~K}$ describe a clear distribution of deep L2 defect states with a maximum value of $N c / \mu=5 \times 10^{10} / \mathrm{cm}^{2} \mathrm{~V} / \mathrm{eV}$. However, 108 days after deposition the MPC curve taken at $270 \mathrm{~K}$ describes a broad and flat plateau with $N c / \mu=2 \times 10^{10} / \mathrm{cm}^{2} \mathrm{~V} / \mathrm{eV}$. Nevertheless, the temporal evolution of the defect state distribution shows no simple picture. On the one hand, MPC detects a lower deep state density, which favors the model of defect annihilation proposed by Ielmini. On the other hand, MPC reveals an increasing density of shallow L1 defects as proposed by Pirovano. ${ }^{14}$ The maximum value of the MPC DOS describing shallow L1 defects increases from 

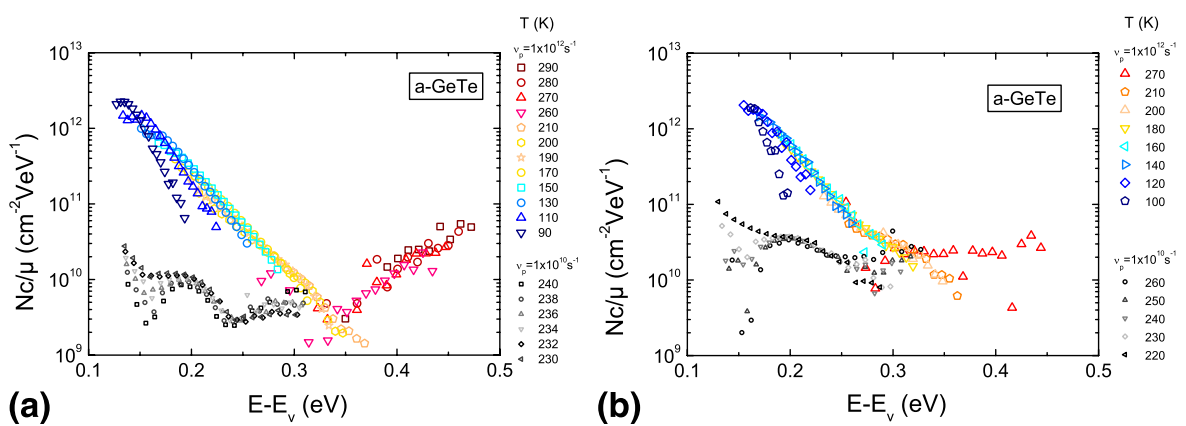

FIG. 5. Defect state densities $N c / \mu$ measured by MPC experiments in the same a-GeTe thin film at different sample ages of 29 days (a) and 108 days (b) after deposition. The MPC spectra shown here are scaled according to Eq. (5) taking the influence of a temperature-dependent band gap into account. The temporal change in defect state density shows no simple picture: Whereas deep in the gap a lower trap state density is detected, the density of shallow L1 defects is observed to increase with time as well as the MPC DOS at $\sim 0.35 \mathrm{eV}$. In contrast, no drastic change in the valence band tail density can be stated. However, the stronger bending of the MPC curves taken from 100 to $180 \mathrm{~K}$ indicates an eventual change of the $\xi$ parameter upon aging which describes the parabolic decrease of the optical band gap with increasing temperature.

$N c / \mu=1 \times 10^{10} / \mathrm{cm}^{2} \mathrm{~V} / \mathrm{eV}$ to $N c / \mu=4 \times 10^{10} / \mathrm{cm}^{2} \mathrm{~V} / \mathrm{eV}$ with increasing sample age. Furthermore, these shallow defect levels are detected in a larger temperature window ranging from 220 to $260 \mathrm{~K}$ at an older sample age, indicating a broadening of the L1 defect distribution. Furthermore, the narrow gap between valence band tail and deep L2 states appearing $\sim 0.35 \mathrm{eV}$ above the valence band edge [see Fig. 5(a)] seems to have disappeared and filled up to a value of $2 \times 10^{10} / \mathrm{cm}^{2} \mathrm{~V} / \mathrm{eV}$ during aging [see Fig. 5(b)]. In contrast with those deep L2 and shallow L1 defect levels the $N c / \mu$ values describing the valence band tail show no significant change. However, at a sample age of 108 days the envelope describing the valence band shows a slight bending around $0.2 \mathrm{eV}$, see Fig. 5(b). This bending may indicate that the $\xi$-value used in the corrected energy scale according to Eq. (5) may have changed slightly upon aging.

The evolution of band tail state densities was studied further by means of PDS, see Fig. 6. This technique enables a sophisticated measurement of the absorption coefficient $\alpha$ as a function of photon energy $E$ of the incident light. Near the optical band gap $\left(E_{\mathrm{g}} \sim 0.8 \mathrm{eV}\right)$ the absorption coefficient shows an exponential dependence:

$$
\alpha(E)=\alpha_{0} \cdot \exp \left(E / E_{\mathrm{u}}\right)
$$

The Urbach energy $E_{\mathrm{u}}$ describing the exponential increase of absorption coefficient reflects the width of the exponentially decaying valence and conduction band tails. ${ }^{36}$ However, even though annealing for $1 \mathrm{~h}$ at $140{ }^{\circ} \mathrm{C}$ shows a significant increase in the amorphous state resistivity no clear change of the Urbach energy $E_{\mathrm{u}}$ can be measured. Together with the MPC results presented in Fig. 5 this finding indicates that neither the valence band nor the conduction band changes upon resistance drift. Even though no change in the Urbach energy $E_{\mathrm{u}}$ could be detected, our PDS measurements clearly demonstrate a shift of $\alpha(E)$ toward higher photon energies. This shift indicates an opening

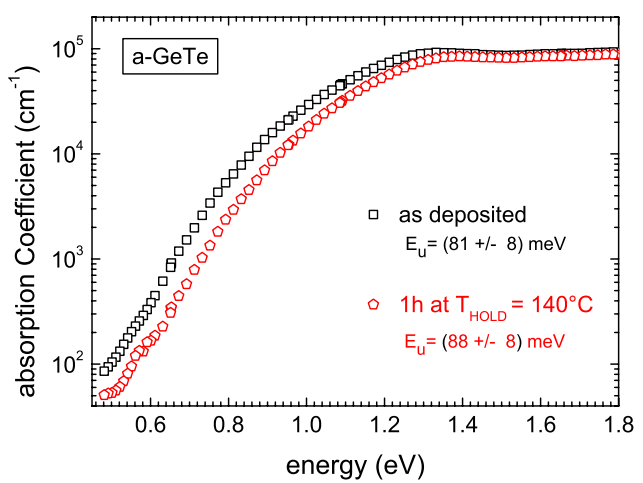

FIG. 6. Absorption coefficient $\alpha(E)$ measured by photothermal spectroscopy (PDS) in amorphous as-deposited and postannealed $\mathrm{a}-\mathrm{GeTe}$ thin films. The postannealed sample has been heated for $1 \mathrm{~h}$ at $T_{\text {HOLD }}=140{ }^{\circ} \mathrm{C}$. In both investigated amorphous samples the absorption coefficient $\alpha$ increases first exponentially with increasing photon energy $E$, i.e., $\alpha(E) \sim \exp \left(E / E_{u}\right)$. Annealing shows no significant influence on the Urbach energy $E_{\mathrm{u}}$, which reflects the width of the valence and conduction band tails. Even though no drastic change in the band tail states upon annealing could be verified by PDS, these measurements reveal a shift of $\alpha(E)$ toward higher photon energies. This shift upon annealing is caused by band gap opening. ${ }^{16}$

of the optical band gap upon annealing. In amorphous materials the optical band gap can be identified with that energy $E_{\mathrm{g}}$ at which the absorption coefficient equals $\alpha(E)=10 \% \mathrm{~cm} .{ }^{37}$ Using this definition of an optical band gap for disordered structures the band gap increases from $E_{\mathrm{g}}=0.85$ to $E_{\mathrm{g}}=0.93 \mathrm{eV}$. This band gap widening has a strong influence on the activation energy of conduction $E_{\mathrm{a}}$ describing the thermally activated behavior of the amorphous state resistivity $\rho$ :

$$
\rho=\rho_{0} \exp \left(E_{\mathrm{a}} / k_{\mathrm{b}} T\right)
$$

Figure 7 compares optical band gaps with activation energies $E_{\mathrm{a}}$ in amorphous-deposited and postannealed $\mathrm{a}-\mathrm{GeTe}$ thin films annealed for $1 \mathrm{~h}$ at the indicated holding temperature $T_{\mathrm{HOLD}}$. Upon annealing the optical band gap 

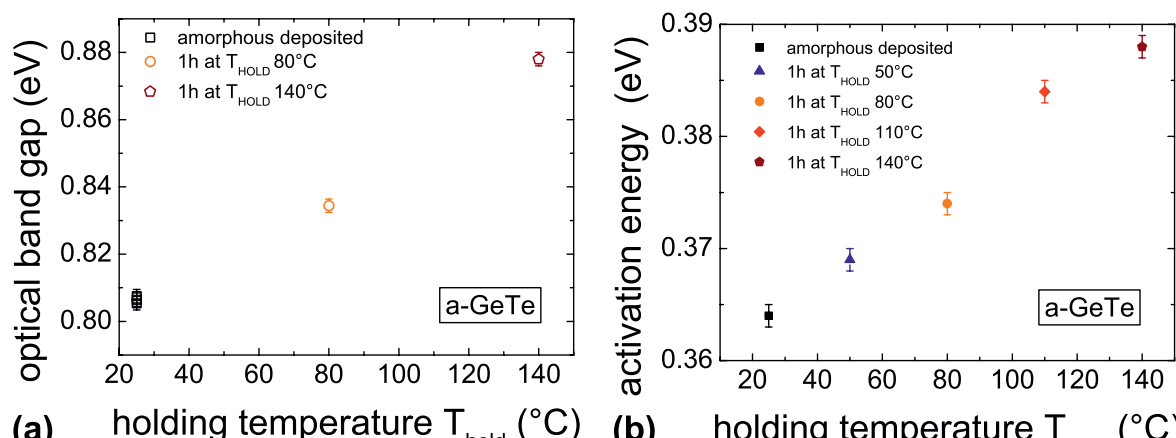

FIG. 7. Optical band gaps $E_{\mathrm{g}}$ and activation energies of conductivity $E_{\mathrm{a}}$ measured in amorphous deposited and postannealed GeTe-thin films heated for $1 \mathrm{~h}$ at the indicated holding temperature $T_{\mathrm{HOLD}}$. Optical band gaps and activation energy increase upon annealing, where the increase is linear with increasing holding temperature $T_{\mathrm{HOLD}}$.

$E_{\mathrm{g}}$ and the activation energy of conduction $E_{\mathrm{a}}$ are observed to increase leading to an increasing amorphous state resistivity according to Eq. (8). These results clearly show the impact of band gap opening on resistance drift. The impact of band gap widening has also been verified for $\mathrm{a}-\mathrm{Ge}_{2} \mathrm{Sb}_{2} \mathrm{Te}_{5}$ in recent studies. ${ }^{38}$

Crystalline and amorphous PCMs demonstrate a very different bonding character. Resonant bonding in their crystalline phase leads to ordering and alignment of $p$-orbitals on adjacent molecular units. In this symmetric atomic arrangement, the valence electrons resonate between bonds of the original cubic lattice. For normal two-center bonds, the matrix element $\mathrm{M}$ is about one bond length. In consequence of resonance bonding the aligned chains yield to $\mathrm{M}$ values of typical two bond lengths giving rise to high transition matrix elements and coordination numbers higher than expected from the 8-N rule. ${ }^{26,39,40}$

However, in disordered PCMs the resonant bonding character breaks down and consequently the band gap in the amorphous phase becomes larger than in the crystalline state due to different extensions of the atomic wave function. Furthermore, Edwards et al. ${ }^{41}$ have shown that the free carrier transport in c-GeTe is determined by the $\mathrm{Ge}$ vacancy. The Ge vacancy is the most easily formed defect in rhombohedral GeTe pinning the Fermi level close to the valence band edge. In contrast the Fermi level in a-GeTe is pinned near the middle of the forbidden energy gap. The Fermi level pinning in amorphous PCMs most probably originates from defects having a negative electron correlation energy, i.e., negative-U, hindering to detect those defects by Electron Spin Resonance.

\section{CONCLUSION}

This work has demonstrated the role of localized defect states on threshold switching and resistance drift phenomena in amorphous PCMs. In chalcogenides with similar optical band gaps a strong correlation between threshold field and trap state density is found: Those alloys which show a high electrical threshold field also show a high density of shallow and deep trap states. This result supports a generation and recombination model of electrical switching. In addition we provide direct experimental proof for a decreasing deep defect concentration as proposed in the Ielmini model. However, no simple evolution of defect state density with time is observed in a-GeTe. Whereas the density of deep states near the Fermi energy decreases, the density of shallow defects increases with increasing sample age. In contrast to the deep L2 and shallow L1 defect levels no drastic change in the band tail densities is observed. Nevertheless, even though band tail state densities remain unchanged the resistance drift effect in a-GeTe is strongly linked to band gap opening.

\section{REFERENCES}

1. M. Wuttig and N. Yamada: Phase-change materials for rewriteable data storage. Nat. Mater. 6, 824 (2007).

2. B.S. Lee, J.R. Abelson, S.G. Bishop, D-H. Kang, B-K. Cheong, and K-B. Kim: Investigation of the optical and electronic properties of $\mathrm{Ge}_{2} \mathrm{Sb}_{2} \mathrm{Te}_{5}$ phase change material in its amorphous, cubic, and hexagonal phases. J. Appl. Phys. 97, 093509 (2005).

3. I. Friedrich, V. Weidenhof, W. Njoroge, P. Franz, and M. Wuttig: Structural transformation of $\mathrm{Ge}_{2} \mathrm{Sb}_{2} \mathrm{Te}_{5}$ films studied by electrical resistance measurements. J. Appl. Phys. 87, 4130 (2000).

4. G. Bruns, P. Merkelbach, C. Schlockermann, M. Salinga, M. Wuttig, T.D. Happ, J.B. Philipp, and M. Kund: Nanosecond switching in GeTe phase change memory cells. Appl. Phys. Lett. 95, 043108 (2009).

5. M.H.R. Lankhorst, B.W.S. Ketelaars, and R.A.M. Wolters: Low-cost and nanoscale non-volatile memory concept for future silicon chips. Nat. Mater. 4, 347 (2005).

6. M. Wuttig: Phase-change materials: Towards a universal memory? Nat. Mater. 4, 265 (2005).

7. S.R. Ovshinsky: Reversible electrical switching phenomena in disordered structures. Phys. Rev. Lett. 21, 1450 (1968).

8. D. Krebs, S. Raoux, C.T. Rettner, G.W. Burr, R.M. Shelby, M. Salinga, C.M. Jefferson, and M. Wuttig: Characterization of phase change memory materials using phase change bridge devices. Appl. Phys. Lett. 106, 054308 (2009).

9. M. Boniardi, A. Redaelli, A. Pirovano, I. Tortorelli, and F. Pellizzer: A physics-based model of electronic conduction decrease with time in amorphous $\mathrm{Ge}_{2} \mathrm{Sb}_{2} \mathrm{Te}_{5}$. J. Appl. Phys. 105, 084506 (2009). 
10. M. Chen, K.A. Rubin, and R.W. Barton: Compound materials for reversible, phase-change optical data storage. Appl. Phys. Lett. 49, 502 (1986).

11. J. Luckas, A. Piarristeguy, G. Bruns, P. Jost, S. Grothe, R. Schmidt, C. Longeaud, and M. Wuttig, Stoichiometry dependence of resistance drift phenomena in amorphous GeSnTe phase-change alloys. J. Appl. Phys. 113, 023704 (2013).

12. D. Ielmini and Y. Zhang: Analytical model for subthreshold conduction and threshold switching in chalcogenide-based memory devices. J. Appl. Phys. 102, 054517 (2007).

13. A. Redaelli, A. Pirovano, A. Benvenuti and A.L. Lacaita: Threshold switching and phase transition numerical models for phase change memory simulations. J. Appl. Phys. 103, 111101 (2008).

14. A. Pirovano, A.L. Lacaita, F. Pellizzer, S.A. Kostylev, A. Benvenuti, and R. Bez: Low-field amorphous state resistivity and threshold voltage drift in chalcogenide materials. IEEE Trans. Electron Devices 51, 714 (2004).

15. D. Ielmini, S. Lavizzari, D. Sharma, and A.L. Lacaita: Temperature acceleration of structural relaxation in amorphous $\mathrm{Ge}_{2} \mathrm{Sb}_{2} \mathrm{Te}_{5}$. Appl. Phys. Lett. 92, 193511 (2008).

16. D. Krebs, R.M. Schmidt, J. Klomfaß, J. Luckas, G. Bruns, C. Schlockermann, M. Salinga, R. Carius, and M. Wuttig: Impact of DoS changes on resistance drift and threshold switching in amorphous phase-change materials. J. Non-Cryst. Solids 358, 2412 (2012).

17. W.B. Jackson, N.M. Amer, A.C. Boccara, and D. Fournier: Photothermal deflection spectroscopy and detection. Appl. Opt. 20, 1333 (1981).

18. H. Oheda: Phase-shift analysis of modulated photocurrent - Its application to the determination of the energetic distribution of gap states. J. Appl. Phys. 52, 6693 (1981).

19. R. Brüggemann, C. Main, J. Berkin, and S. Reynolds: An evaluation of phase-shift analysis of modulated photocurrents. Philos. Mag. B 62, 29 (1990).

20. C. Longeaud and J. P. Kleider: General-analysis of the modulatedphotocurrent experiment including the contributions of holes and electrons. Phys. Rev. B 45, 11672 (1992).

21. J. Luckas, D. Krebs, M. Salinga, M. Wuttig, and C. Longeaud: Investigation of defect states in the amorphous phase of phase change alloys $\mathrm{GeTe}$ and $\mathrm{Ge}_{2} \mathrm{Sb}_{2} \mathrm{Te}_{5}$. Phys. Status Solidi C 7, 852 (2010).

22. N. Nagaosa, J. Sinova, S. Onoda, A.H. MacDonald, and N.P. Ong: Anomalous Hall effect. Rev. Mod. Phys. 82, 1539-1592 (2010).

23. J. Luckas, S. Kremers, D. Krebs, M. Salinga, M. Wuttig, and C. Longeaud: The influence of a temperature dependent band gap on the energy scale of modulated photocurrent experiments. J. Appl. Phys. 110, 013719 (2011).

24. S. Raoux, D. Cabrera, A. Devasia, S. Kurinec, H. Cheng, Y. Zhu, C. Breslin, J. Jordan-Sweet, C.T. Rettner, G.W. Burr, M. Salinga, and M. Wuttig: Influence of dopants on the crystallization temperature, crystal structure, resistance, and threshold field for $\mathrm{Ge}_{2} \mathrm{Sb}_{2} \mathrm{Te}_{5}$ and GeTe phase change materials. E/PCOS (2011).
25. D. Krebs, S. Raoux, C.T. Rettner, G.W. Burr, M. Salinga, and M. Wuttig: Threshold field of phase change memory materials measured using phase change bridge device. Appl. Phys. Lett. 95, 082101 (2009).

26. S. Shportko, S. Kremers, M. Woda, D. Lencer, J. Robertson, and M. Wuttig: Resonant bonding in crystalline phase-change materials. Nat. Mater. 7, 653 (2008).

27. S. Kremers: Optische Eigenschaften von Phasenwechselmaterialien für zukünftige optische und elektronische Speicheranwendungen. Ph.D. Thesis, RWTH University, Aachen, Germany, 2009.

28. C. Longeaud and S. Tobbeche: The influence of hopping on modulated photoconductivity. J. Phys. Condens. Matter. 21, 045508 (2009)

29. W. Shockley and W.T. Read: Statistics of the recombinations of holes and electrons. Phys. Rev. B 87, 835 (1952).

30. J.G. Simmons and G.W. Taylor: Nonequilibrium steady-state statistics and associated effects for insulators and semiconductors containing an arbitrary distribution of traps. Phys. Rev. B 4, 502 (1971).

31. M.H. Cohen, H. Fritzsche, and S.R. Ovshinsky: Simple band model for amorphous semiconducting alloys. Phys. Rev. Lett. 22, 1065 (1969).

32. M. Kastner, D. Adler, and H. Fritzsche: Valence-alternation model for localized gap states in lone-pair semiconductors. Phys. Rev. Lett. 37, 1504 (1976).

33. D. Adler, M.S. Shur, M. Silver, and S.R. Ovshinsky: Threshold switching in chalcogenide-glass thin-films. J. Appl. Phys. 51, 3289 (1980).

34. K. Jandieri, O. Rubel, S. Baranovski, A. Reznik, J. Rowlands, and S.O Kasap: Lucky-drift model for impact ionization in amorphous semiconductors. J. Mater. Sci. Mater. Electron. 20, 221 (2008).

35. I.V. Karpov, M. Mitra, D. Kau, G. Spadini, Y.A. Kryokov, and V.G. Karpov: Fundamental drift of parameters in chalcogenide phase change memory. J. Appl. Phys. 102, 124503 (2007).

36. S. John, C. Soukhoulis, M.H. Cohen, and E.N. Economou: Theory of electron band tails and the Urbach optical-absorption edge. Phys. Rev. Lett. 57, 1777 (1986).

37. J. Stuke: Review of optical and electrical properties of amorphous semiconductors. J. Non-Cryst. Solids 4, 1 (1970).

38. P. Fantini, S. Brazzelli, E. Cazzini, and A. Mani: Band gap widening with time induced by structural relaxation in $\mathrm{Ge}_{2} \mathrm{Sb}_{2} \mathrm{Te}_{5}$ films. Appl. Phys. Lett. 100, 013505 (2012).

39. D. Lencer, M. Salinga, and M. Wuttig: Design rules for phasechange materials in data storage applications. Adv. Mater. 23, 2030 (2011).

40. B. Huang and J. Robertson: Bonding origin of optical contrast in phasechange memory materials. Phys Rev B 81, 081204 (2010).

41. A.H. Edwards, A.C. Pineda, P.A. Schultz, M.G. Martin, A.P. Thompson, and H.P. Hjalmarson: Theory of persistent, p-type, metallic conduction in c-GeTe. J. Phys. Condens. Matter. 17, L329 (2005). 